\title{
Universality and size effects in the Barkhausen noise
}

Cite as: Journal of Applied Physics 87, 7031 (2000); https://doi.org/10.1063/1.372921

Published Online: 28 April 2000

Gianfranco Durin, and Stefano Zapperi

\section{ARTICLES YOU MAY BE INTERESTED IN}

Scaling aspects of domain wall dynamics and Barkhausen effect in ferromagnetic materials Journal of Applied Physics 75, 5490 (1994); https://doi.org/10.1063/1.355666

Domain-wall dynamics and Barkhausen effect in metallic ferromagnetic materials. I. Theory Journal of Applied Physics 68, 2901 (1990); https://doi.org/10.1063/1.346423

Barkhausen noise in soft amorphous magnetic materials under applied stress

Journal of Applied Physics 85, 5196 (1999); https://doi.org/10.1063/1.369122

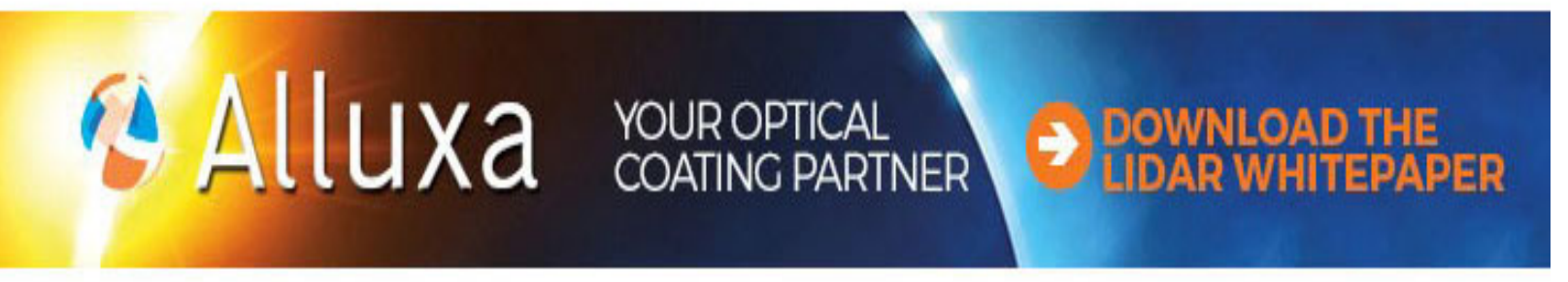




\title{
Universality and size effects in the Barkhausen noise
}

\author{
Gianfranco Durin ${ }^{\text {a) }}$ \\ Istituto Elettrotecnico Nazionale Galileo Ferraris and INFM, Corso M. d'Azeglio 42, I-10125 Torino, Italy \\ Stefano Zapperib) \\ INFM Sezione di Roma 1, Departimento di Fisica, Università “La Sapienza," P. le A. Moro 200185 \\ Roma, Italy
}

We show that the Barkhausen avalanches exhibit power law distributions and scaling exponents belonging to two distinct universality classes. We explain these results in terms of the critical behavior of the domain wall at the depinning transition, with exponents set by the long-range dipolar interactions arising from local magnetostatic fields, and by the elastic curvature of the wall. We are also able to predict the precise dependence of the cutoff on the demagnetizing factor $k$ due to sample size. These predictions are experimentally confirmed on three samples (two polycrystalline $6.5 \mathrm{wt} \%$ $\mathrm{Si}-\mathrm{Fe}$ and an amorphous $\mathrm{Fe}_{21} \mathrm{Co}_{64} \mathrm{~B}_{15}$ under applied tensile stress) which are progressively cut in order to increase $k$. All these results allow us to link the material microstructure and the sample geometry to the macroscopic noise properties. (C) 2000 American Institute of Physics.

[S0021-8979(00)70608-6]

\section{INTRODUCTION}

Since its discovery, the Barkhausen effect has been recognized as a promising tool to investigate the microscopic magnetization processes along the hysteresis loop in soft magnetic materials. A definite link between the observed statistical properties of the Barkhausen signal and the specific magnetization details, together with information on material microstructure, has been only partially established, mainly using phenomenological approaches and on a selected range of magnetic materials. ${ }^{1,2}$ Undoubtedly, this goal is complicated by the intrinsic complexity of the phenomenon, which justifies the about 80 years of studies. For a long time and still recently, most of the studies have been focused on the properties of the power spectra. Using pure phenomenological approaches, the signal was treated as a statistical superposition of uncorrelated elementary jumps, without any direct relation with microscopic magnetization events. Effectively, power spectra appear too complex to be easily explained even by the recent most successful theories, as the information they contain is an average over different spacetime processes, which require high order statistics to be analyzed in detail. ${ }^{3}$ In addition, power spectra do not display any universal property, contrary to the distributions of duration and size of Barkhausen jumps, extensively studied in the recent literature. ${ }^{4-6}$ Universality implies that the scaling exponents do not depend on the microscopic details of the system, but only on the range of relevant fields governing the domain wall dynamics and on the dimensionality of the system.

In this context, we have recently proposed ${ }^{7,8}$ a microscopic model where universality and criticality are understood in terms of depinning transition of the domain wall. Using a detailed description of the relevant interaction fields acting on the domain wall, we came to the conclusion that

\footnotetext{
${ }^{a)}$ Electronic mail: durin@omega.ien.it

${ }^{b)}$ Electronic mail: zapperi@pil.phys.uniroma1.it
}

two possible classes of universality exist exhibiting different critical exponents. These different classes are a consequence of the competition between long-range local magnetostatic fields arising from magnetic charges in a deformed domain wll and the short-range surface tension. In general, soft magnetic materials with grain structure exhibit the long-range universality class, while amorphous systems under applied tensile stress and high anisotropic materials belong to the short-range class. ${ }^{4,6,9,10}$ The theoretical and experimental demonstration of this behavior has an important consequence, as it is thus possible to investigate the effect of the finite size of the system on the observed properties of the Barkhausen signal. In particular, the sample geometry and the microstructure (grains, quenched-in stress, etc.) should in principle affect the behavior of cutoff of the power law distributions. ${ }^{11}$ In this paper, after a short review of recent results, we show how the cutoff can be theoretically calculated and present the simulations and the experimental data confirming these results. Finally, we give indications on how this approach can be used to further explore the microstructure of the system.

\section{UNIVERSALITY AND CHARACTERISTIC SIZES}

We assume to have a single $180^{\circ}$ domain wall dividing two regions of opposite magnetization directed along the $x$ axis, and described by its position $h(\mathbf{r})$. The dynamical equation is constructed considering all the contributions to the total energy giving 7,8

$$
\begin{aligned}
\Gamma \frac{\partial h(\mathbf{r}, t)}{\partial t}= & 2 \mu_{0} M_{s} H-k \int d^{d} r^{\prime} h\left(\mathbf{r}^{\prime}, t\right)+\gamma_{w} \nabla^{2} h(\mathbf{r}, t) \\
& +\int d^{d} r^{\prime} J\left(\mathbf{r}-\mathbf{r}^{\prime}\right)\left(h\left(\mathbf{r}^{\prime}\right)-h(\mathbf{r})\right)+\eta(\mathbf{r}, h),
\end{aligned}
$$

where we have considered a generic $d$-dimensional domain wall. We indicate with $\Gamma$ the viscosity, and with $M_{s}$ the 
TABLE I. Critical exponents as obtained by renormalization group calculations (Ref. 12) and scaling relations (Ref. 8) as a function of the dimension $d$ of the domain wall and the range $\mu$ of iterations controlling the domain wall dynamics.

\begin{tabular}{lcc}
\hline \hline Correlation length & $\nu$ & $3 /(\mu+d)$ \\
Roughness & $\zeta$ & $(2 \mu-d) / 3$ \\
Dynamical & $z$ & $\mu-(4 \mu-2 d) / 9$ \\
Size distribution & $\tau$ & $2-3 \mu /[2(d+\mu)]$ \\
Duration distribution & $\alpha$ & $(2 \mu+8 d) /(5 \mu+2 d)$ \\
\hline \hline
\end{tabular}

saturation magnetization. The different terms on the righthand side come, respectively, from the pressure exerted on the wall by the applied field $H$, the demagnetizing counterfield $-k M$, the wall curvature with surface tension $\gamma_{w}$, and from dipolar interactions due to local magnetic charges, with a kernel $J$ given by

$$
J\left(\mathbf{r}-\mathbf{r}^{\prime}\right)=\frac{\mu_{0} M_{s}^{2}}{2 \pi\left|\mathbf{r}-\mathbf{r}^{\prime}\right|^{3}}\left(1+\frac{3\left(x-x^{\prime}\right)^{2}}{\left|\mathbf{r}-\mathbf{r}^{\prime}\right|^{2}}\right) .
$$

The last term $\eta(\mathbf{r}, h)$ is a Gaussian uncorrelated random field, of amplitude $D \equiv \sqrt{\left\langle\eta^{2}\right\rangle}$, taking into account all the possible effects of dislocations, residual stress and nonmagnetic inclusions.

The critical behavior of Eq. (1) is ruled by dipolar interactions which are more relevant than the surface tension at large length scales. However, if the surface tension is very large, we may effectively see a different critical behavior. In general, for a kernel of the type $J(q)=J_{0}|q|^{\mu}$ in Fourier space ( $\mu=1$ corresponds to dipolar interactions and $\mu=2$ to the surface tension), the critical dimension turns out to be $d_{c}=2 \mu$, so that three-dimensional samples (i.e., $d=2$ ) with strong dipolar interactions display mean field properties. ${ }^{7,8}$ In general, critical exponents depend only on the values of the dimension $d$ and on $\mu$, while the cutoff is set by the demagnetizing factor $k$ and the amplitude $D$, as we discuss below.

When the restoring force is negligible, Eq. (1) displays a depinning transition as a function of the applied field $H .^{12,13}$ This means that the domain wall moves only if the applied field overcomes a critical field $H_{c}$. Close to the depinning transition, the domain wall proceeds with avalanches whose sizes $s$ are distributed as

$$
P(s) \sim s^{-\tau} f\left(s / s_{0}\right),
$$

where $s_{0}$ is the distribution cutoff, which diverges as $s_{0}$ $\sim\left(H-H_{c}\right)^{-1 / \sigma}$. There is a natural correlation length $\xi$ associated with this cutoff size $s_{0}$. In general, a portion of domain wall of area $\sim \xi^{d}$ moves for a displacement scaling as $D \xi^{\zeta}$, where $\zeta$ is the roughness exponent, so that $s_{0}$ $\sim D \xi^{d+\zeta}$. Also the correlation length must diverge at $H_{c}$ as $\xi \sim\left(H-H_{c}\right)^{-\nu}$, where $\nu$ is the correlation length exponent. Following analogous considerations, the duration $T$ of avalanches, distributed as $P(T) \sim T^{-\alpha} g\left(T / T_{0}\right)$ has a cutoff $T_{0}$ diverging as $T_{0} \sim\left(H-H_{c}\right)^{-1 / \tilde{\sigma}} \sim D \xi^{z}$, where $z$ is the dynamical exponent. All these critical exponents have been calculated using renormalization group methods ${ }^{7,12}$ (Table I).

The presence of the demagnetizing term in Eq. (1) has the effect of keeping the wall close to the depinning transition, without changing the values of the critical exponents.
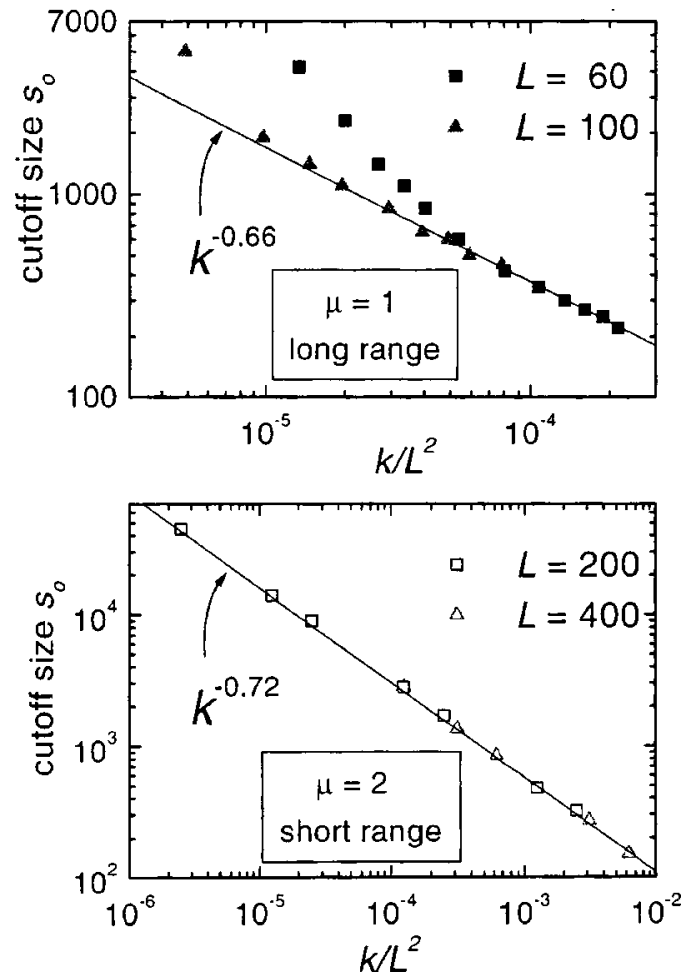

FIG. 1. Simulation results for the cutoff $s_{o}$ of the jump size distribution using Eq. (1). The two universality classes correspond to a kernel in the equation of the $|q|^{\mu}$ type in Fourier space, with $\mu=1$ (long range dipolar interactions, up), and $\mu=2$ (short range elastic interactions, down).

On the other hand, it affects the cutoff values of the distributions, as it reduces the net field applied to the domain wall, and consequently, the maximum size (and duration) of an avalanche. This effect can be estimated considering that the maximum net field applied to the wall $H-H_{c}$ must be of the order of the variation of the demagnetizing field for the biggest jump, that is, $k \Delta M \sim k s_{0}$. This gives $\xi \sim\left(H-H_{c}\right)^{-\nu}$ $\sim\left(k s_{0}\right)^{-\nu} \sim k^{-\nu} \xi^{-\nu(d+\zeta)}$, so that, using $\mu=1 / \nu+\zeta$

$$
\xi \sim k^{-\nu_{k}}, \quad \nu_{k}=1 /(\mu+d) \text {. }
$$

Consequently, the dependence of the cutoffs $s_{0}$ and $T_{0}$ on the demagnetizing factor $k$ turns out to be

$$
\begin{aligned}
& s_{0} \sim D\left(k / J_{0}\right)^{-1 / \sigma_{k},} \quad 1 / \sigma_{k}=\nu_{k}(d+\zeta), \\
& T_{0} \sim D\left(k / J_{0}\right)^{-\Delta_{k},} \quad \Delta_{k}=z \nu_{k} .
\end{aligned}
$$

Inserting the results of the renormalization group calculations (Table I), we obtain, for $d=2,1 / \sigma_{k}=2 / 3$ independent of $\mu$, while $\Delta_{k}=1 / 3$ for $\mu=1$, and $\Delta_{k}=7 / 18$ for $\mu=2$.

\section{SIMULATIONS AND EXPERIMENTAL RESULTS}

To verify the theoretical predictions given above, we performed a series of simulations using an automaton version $(\Delta h / \Delta t=0,1)$ of Eq. (1). In Fig. 1 we present the results of the exponents $1 / \sigma_{k}$ for both classes in $d=2$, showing the good agreement with the theoretical predictions. Clearly, the value of the demagnetizing factor $k$ must be chosen to give a maximum area of the domain wall $\xi^{d}$ that is much smaller than the size $L^{d}$ of the system. 
We also performed a series of experimental characterization (see Refs. 8 and 14 for details) of the Barkhausen signal on three different kinds of samples belonging to the different classes; two polycrystalline $\mathrm{Fe}-\mathrm{Si} 6.5 \mathrm{wt} \%$ alloys annealead at $1050^{\circ} \mathrm{C}$ and $1200{ }^{\circ} \mathrm{C}$ to get different grain sizes, ${ }^{11}$ and a magnetostrictive $\left(\lambda_{s} \simeq 35 \times 10^{-6}\right)$ amorphous $\mathrm{Fe}_{21} \mathrm{Co}_{64} \mathrm{~B}_{15}$ alloy. This material has been measured after the application of a tensile stress of about $100 \mathrm{MPa}$. The former kind of materials shows a critical exponent $\tau \sim 1.5$, while the latter has $\tau \sim 1.27$, so that they correspond to the long range class $(\mu=1)$ and the short range one $(\mu=2)$, respectively (see Refs. 8-10). In order to evaluate the influence of the geometry on the demagnetizing factor, we choose to progressively shorten the same sample, and measure the Barkhausen signal always in the same cross section in the middle of the ribbon. This uniformity of condition of measurements would not be fulfilled using different samples which would show, at least, different domain patters. Effectively, the range of available lengths is quite limited as in samples that are too short (say, less than 6-8 cm) the variation of the demagnetizing field along the ribbon completely changes the domain pattern. This implies getting a variation of permeability of no more than one order of magnitude. The demagnetizing factor is estimated in the usual way. Using a magnetic yoke, we estimate the intrinsic permeability $\mu_{\text {int }}$, and for each sample we measure the apparent permeability $\mu_{\text {app }}$, so that $k=\mu_{\text {app }}$ $-\mu_{\text {int }}$.

Experimental results are shown in Fig. 2. Distribution data are analyzed using the same fitting function for all the lengths of a sample considered, to get the estimation of the cutoff values. In particular, for the $\mathrm{Fe}-\mathrm{Si}$ alloys, the cutoff $s_{0}$ decreases upon lowering the applied field frequency, so that an extrapolation to zero frequency is needed. The experimental exponents agree reasonably well with the predictions, and in particular with simulation results, despite various sources of errors, such as the experimental noise, the correct estimation of $k$, and the extrapolation to zero frequency of $s_{0}$. The $\mathrm{Fe}-\mathrm{Si}$ alloy annealead at $1050^{\circ} \mathrm{C}$ shows an interesting effect at low values of the demagnetizing factor, as it clearly deviates from the theoretical behavior, keeping a constant value. This fact can be related to some characteristic length scales of the system, due to the details of the microstructure (especially the grain size) and/or to ribbon thickness. ${ }^{11}$ As seen, in the theory proposed here, the only characteristic length scales are the correlation length controlled by the demagnetizing factor, and the size $L$ of the system, as the disorder is assumed to be an uncorrelated random field. Therefore, we expect that a deviation from the predicted behavior should be ascribed only to the correlation length $\xi$ approaching the smaller geometrical dimension of the system, that is, the ribbon thickness. A detailed analysis shows instead a closer connection to the average grain size. ${ }^{11}$ In any case, the introduction of an appropriate description of the intrinsic microstructure of a material, as in these polycrystallines, will help to clarify this important point.

To summarize, we have shown that the equation of motion of a single domain wall is able to describe experimental data for different types of materials, despite their different microstructure, geometry, internal stresses, and multiple do-
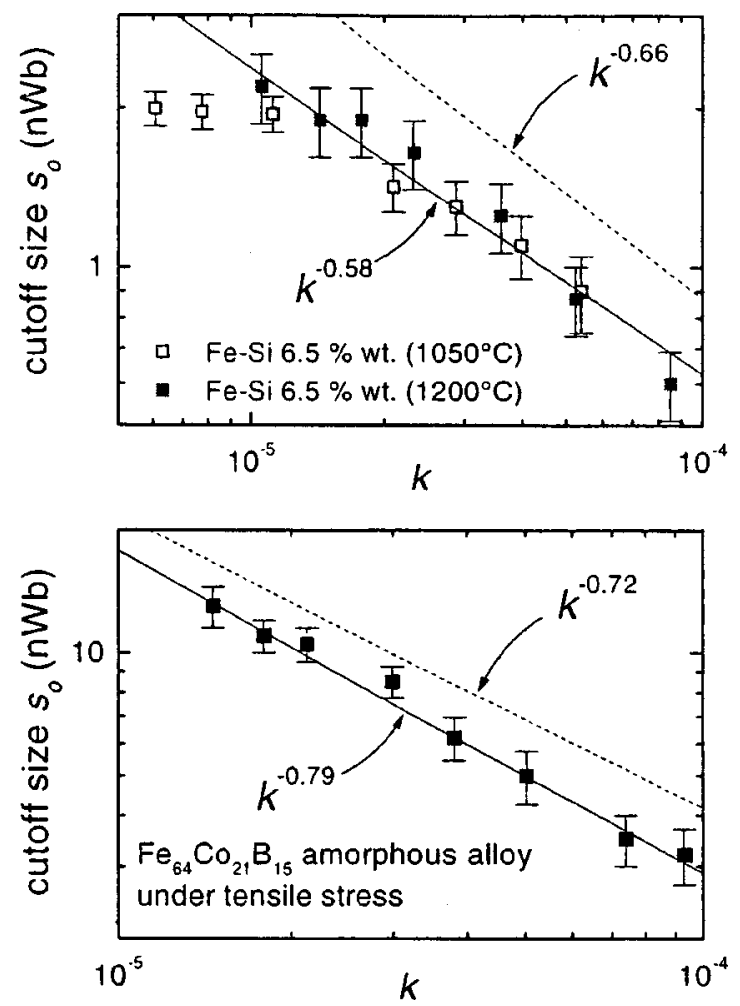

FIG. 2. Experimental results for the cutoff size $s_{0}$ for samples belonging to different universality classes; (up) two polycrystallines $6.5 \mathrm{wt}$ \% $\mathrm{Si}-\mathrm{Fe}$ alloys $(\mu=1)$, and (down) a magnetostrictive amorphous $\mathrm{Fe}_{21} \mathrm{Co}_{64} \mathrm{~B}_{15}$ alloy under tensile stress $(\mu=2)$. Best fit of data is given by continuous lines. Theoretical predictions (dotted lines) are shown for comparison.

main pattern. In particular, we were able to calculate the dependence of the cutoffs of the power law distributions on the demagnetizing field due to the sample geometry. The validity of this approach gives important indications to establish a link between the observed properties of the Barkhausen noise and the material microstructure and sample geometry.

${ }^{1}$ G. Bertotti and A. Montorsi, J. Magn. Magn. Mater. 86, 214 (1990); B. Alessandro, C. Beatrice, G. Bertotti, and A. Montorsi, J. Appl. Phys. 68, 2901 (1990); 68, 2908 (1990).

${ }^{2}$ M. J. Sablik, J. Appl. Phys. 74, 5898 (1993).

${ }^{3}$ J. R. Petta, M. B. Weissman, and G. Durin, Phys. Rev. E 57, 6363 (1998); IEEE Trans. Magn. 34, 1171 (1998).

${ }^{4}$ J. S. Urbach, R. C. Madison, and J. T. Markert, Phys. Rev. Lett. 75, 276 (1995).

${ }^{5}$ D. Spasojevic, S. Bukvic, S. Milosevic, and H. E. Stanley, Phys. Rev. E 77, 2531 (1996)

${ }^{6}$ M. Bahiana et al., Phys. Rev. E 59, 3884 (1999).

${ }^{7}$ P. Cizeau, S. Zapperi, G. Durin, and H. E. Stanley, Phys. Rev. Lett. 79, 4669 (1997).

${ }^{8}$ S. Zapperi, P. Cizeau, G. Durin, and H. E. Stanley, Phys. Rev. B 58, 6353 (1998).

${ }^{9}$ G. Durin, and S. Zapperi, Phys. Rev. Lett (to be published).

${ }^{10}$ G. Durin and S. Zapperi, J. Appl. Phys. 85, 5196 (1999).

${ }^{11}$ G. Durin, C. Beatrice, C. Appino, V. Basso, and G. Bertotti (these proceedings).

${ }^{12}$ T. Nattermann, S. Stepanow, L. H. Tang, and H. Leschhorn, J. Phys. II 2 , 1483 (1992); O. Narayan and D. S. Fisher, Phys. Rev. B 48, 7030 (1993); H. Leschhorn, T. Nattermann, S. Stepanow, and L. H. Tang, Ann. Physik 6, 1 (1997).

${ }^{13}$ H. Ji and M. O. Robbins, Phys. Rev. A 44, 2538 (1991).

${ }^{14}$ G. Durin, A. Magni, and G. Bertotti, Fractals 3, 351 (1995). 